\title{
Lyme Carditis Presenting as Sinus Node Dysfunction and Accelerated Junctional Rhythm
}

Saima Karim ${ }^{1 *}$, Roy Arjoon², B. Julie He$^{2}$, Lynda Rosenfeld ${ }^{2}$, Paras Bhatt ${ }^{2}$

${ }^{1}$ Heart and Vascular Institute, Case Western Reserve University/MetroHealth Medical Center Campus, 2500 Metrohealth Medical Center, Cleveland, Ohio

${ }^{2}$ Section of Cardiovascular Medicine, Yale University School of Medicine, 789 Howard Avenue, Dana 3, New Haven, CT 06510

*Corresponding Author: Saima Karim, DO Metro Health Medical Center 2500 Metro Health Drive Cleveland, OH 44109 Received Date: November 11, 2020; Accepted Date: November 20, 2020; Published Date: November 25, 2020

Citation: Saima Karim., Roy Arjoon., Julie He B., Lynda Rosenfeld., Paras Bhatt., (2020) Lyme Carditis Presenting as Sinus Node Dysfunction and Accelerated Junctional Rhythm. J. Clinical Cardiology and Cardiovascular Interventions, 3(12); Doi:10.31579/2641-0419/106

Copyright: () 2020 Saima Karim, This is an open-access article distributed under the terms of the Creative Commons Attribution License, which permits unrestricted use, distribution, and reproduction in any medium, provided the original author and source are credited.

\begin{abstract}
:
Lyme disease can have cardiac involvement and can subsequently present with various types of atrio ventricular (AV) block. Sinus node dysfunction (SND) and accelerated junctional rhythm are highlighted in this case as an uncommon presentation for Lyme Carditis. This case highlights the importance of having a high index of suspicion for cardiac involvement with Lyme disease when atypical arrhythmias are present.
\end{abstract}

Short title: Sinus Node Dysfunction with Lyme Disease

Key words: atrio ventricular; lyme carditis; lyme disease

\section{Introduction:}

Cardiac involvement in Lyme disease, the most common vector-borne illness in the United States and Europe, usually presents as various degrees of atrioventricular (AV) block (1-3). We present a case of sinus node dysfunction (SND) and accelerated junctional rhythm to emphasize the importance of thinking about this diagnosis in the setting of rhythm disorders other than AV block in appropriate patients.

\section{Case Report:}

A fifty four year old female with a past medical history of diabetes mellitus, Graves disease treated with tapazole, asthma, hyperlipidemia and obesity presented with the new onset dyspnea on exertion. ECG on presentation showed an accelerated junctional rhythm with retrograde $\mathrm{P}$ waves causing enterance block into the sinus node with occasional sinus versus premature atrial complexes with normal AV conduction time (Figure 1).

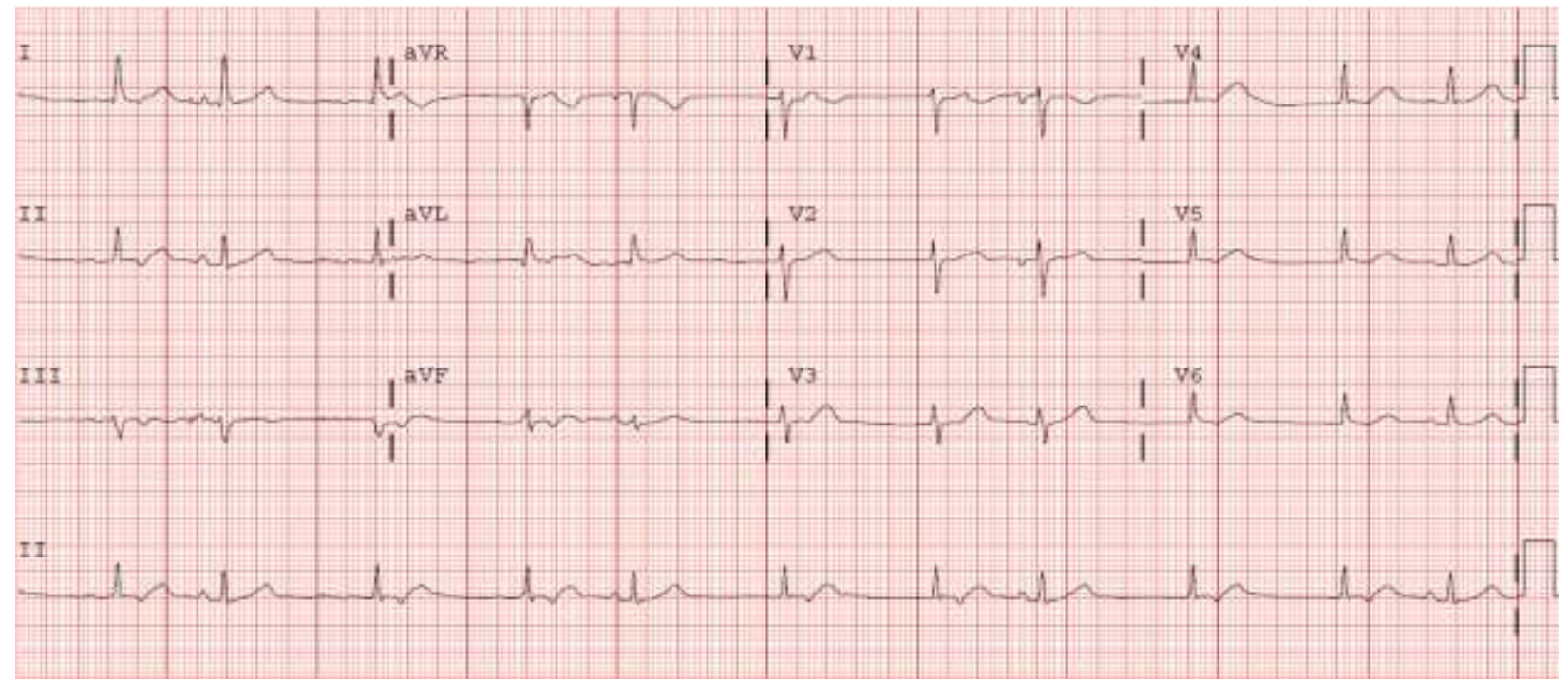

Figure 1: ECG on presentation shows junctional rhythm in the 60s with intermittent organized atrial activity preceding QRS, could be sinus versus ectopic atrial rhythm that conducted with a normal PR interval indicating normal AV conduction. The retrograde $P$ waves from the junctional rhythm result in entrance block to the sinus node. 
Cardiac monitoring demonstrated sinus rhythm versus ectopic atrial rhythm followed by a significant pause and then a junctional escape rhythm (Figure 2).

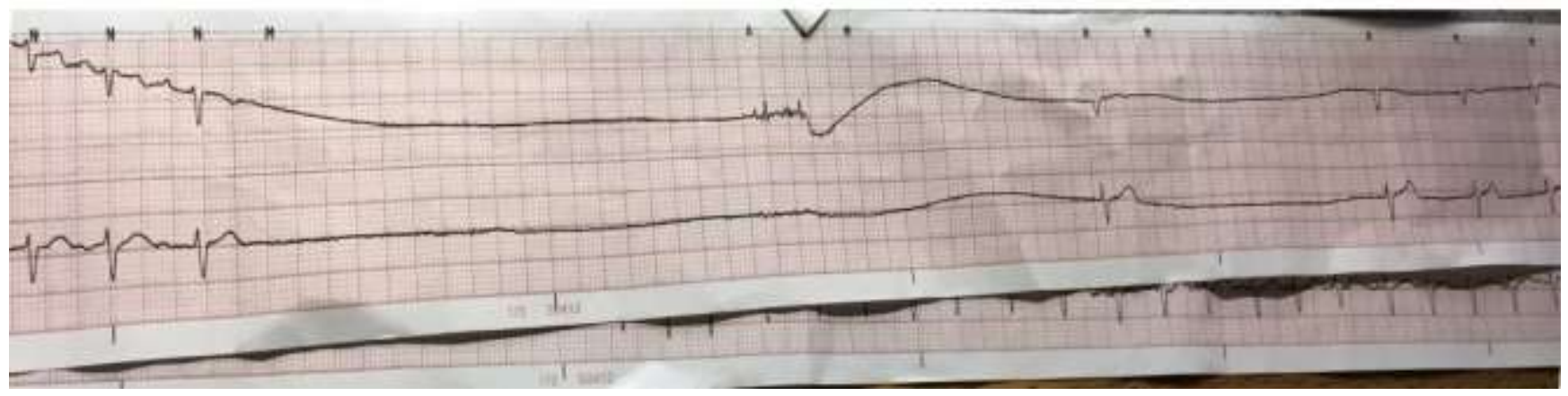

Figure 2: Rhythm shortly after upon presentation with sinus rhythm versus atrial arrhythmia followed by a protracted pause with a junctional escape beat followed by junctional escape rhythm in the setting of Lyme carditis.

The primary pause was 7.2 seconds with a junctional escape complex, but the overall sinus pause was 10.4 seconds (including the secondary pause of 3.2 seconds).

The patient had gone camping one and half weeks prior to admission. She was not aware of any insect bites but developed progressively worsening dyspnea on walking 100 feet a few days later leading her to present to the Emergency Department. She did describe a rash on her left shoulder and abdomen. She denied any edema, chest pain or lightheadedness.

On presentation, her vital signs were stable with a heart rate of 62 beats per minute. Her physical examination revealed tachypnea, bibasilar rales, an irregular rhythm, and a bullseye rash on her lower abdomen. Laboratory evaluation included a normal basic metabolic panel and complete blood count, but an elevated BNP (3740 pg/ml) and CRP (10 $\mathrm{mg} / \mathrm{L})$. A transthoracic echocardiogram demonstrated a left ventricular ejection fraction of $68 \%$ with mild mitral and aortic regurgitation. A contrast CT scan to rule out a pulmonary embolism and she was admitted to the medical service.

Given her history and presentation, B burgdorferi IgM and IgG antibodies were obtained (positive). A western blot showed the B burgdorferi antibody to be 7.51 Lyme Index. She remained on telemetry with daily ECGs and intravenous ceftriaxone was initiated. Within 24 hours of initiation of antibiotics she had intermittent competing junctional rhythm with retrograde conduction and a higher frequency of premature atrial or sinus complexes with normal anterograde AV conduction (Figure 3). At 48 hours, there were more conducted sinus or premature atrial complexes with normal AV conduction with competing junctional beats. At 72 hours, sinus rhythm was predominant. By the fourth day of antibiotic therapy, sinus rhythm with normal conduction overtook the junctional rhythm (Figure 3).

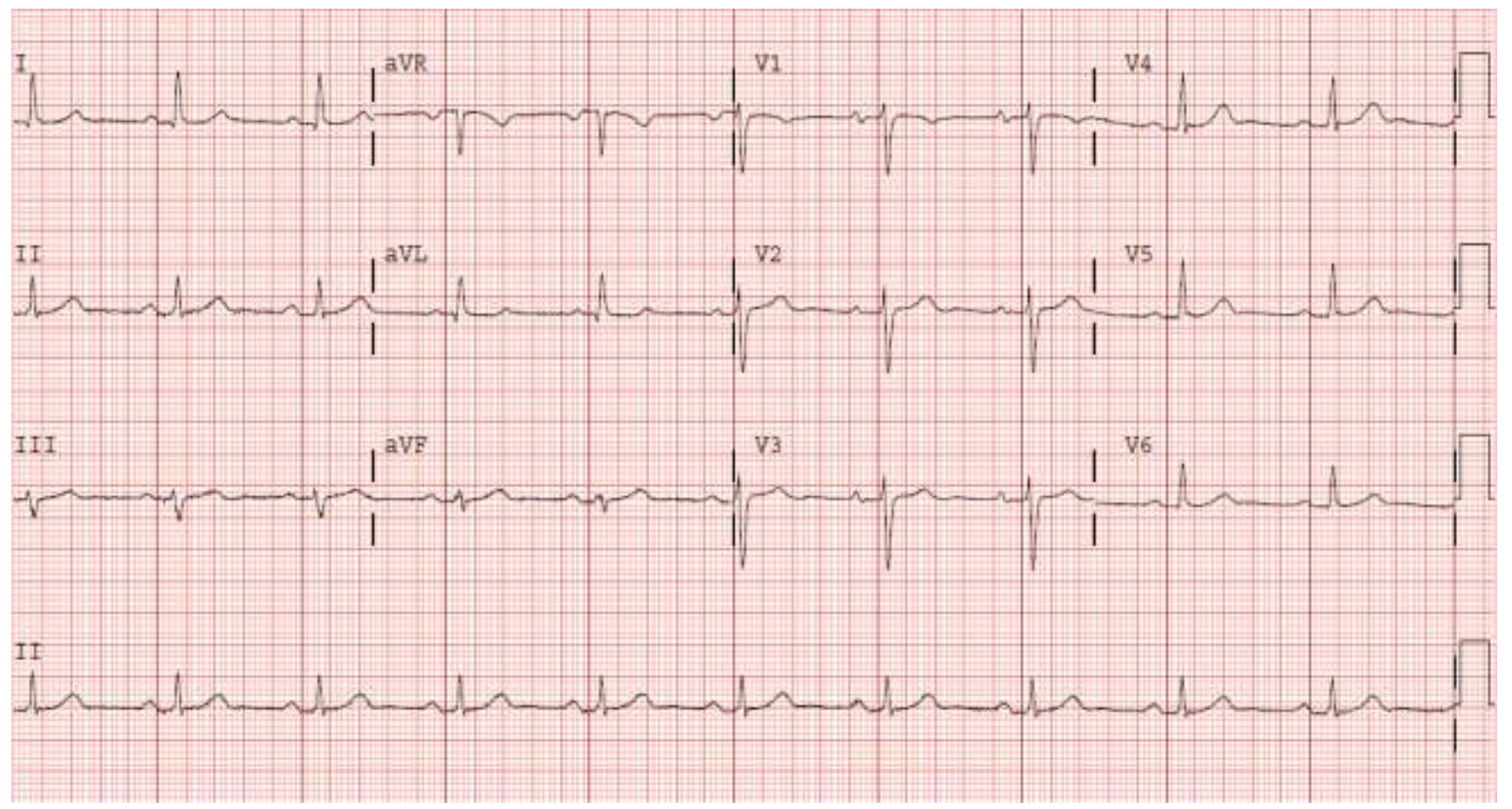

Figure 3: Return of normal sinus rhythm with normal AV conduction on the fourth day of antibiotic therapy.

She had no further sinus pauses. On follow up, the patient continued to have sinus rhythm with no further dyspnea on exertion or signs of sinus node dysfunction on ECG. 


\section{Discussion:}

While cardiac manifestations of Lyme disease including conduction system defects such as AV nodal block, bundle branch block, intraventricular conduction defects, as well as supraventricular arrhythmias and ventricular arrhythmias are well known, sinus node dysfunction with an accelerated junctional rhythm and normal AV conduction is a rarely described phenomenon that has only been highlighted in case reports. The abnormalities of Lyme disease are selflimited and thought to be directly related to the inflammatory response to spirochetes within the cardiac tissue. $(1,2,4-9)$ In the United States the causative agent is the spirochete Borrelia burgdoferi and in Europe, it may be caused by various spirochetes, including B. afzelii, B. garinii, B. burgdorferi, and other related species [2]. Clinical manifestations of Lyme disease include erythema migrans, arthritis, neuritis, and carditis. Lyme carditis typically occurs weeks to months after the onset of the pathognomic erythema migrans rash during the early disseminated stage of the disease. Between $1 \%$ and $10 \%$ of patients with untreated Lyme disease in the United States develop carditis; however, the incidence in Europe appears lower at $0.3 \%$ to $4 \%$ [3].

A literature search reveals only 3 prior case reports of acute sinus node dysfunction in a setting of confirmed Lyme disease, 1 in the United States and 2 in Europe. [6-8]. While the patient above recovered from sinus node dysfunction and accelerated junctional rhythm with antibiotic therapy, a European case report has shown the need for a pacemaker in a 42 year man who was suspected to have infection by B burgdorferi. [8] Another European case report did demonstrate recovery of sinus node function that presented as sinus arrest after treatment with antibiotic for presumed Lyme disease. [6] A North American case report did demonstrate sinus pauses that improved with intravenous ceftriaxone, but the hospital course was protracted lasting 14 days with persistent first degree AV block at the time of discharge. [7] While the incidence of sinus node dysfunction in Lyme disease appears to be relatively low, its recognition is important in the optimal management of the patient. Akin to the therapy for AV nodal conduction problems in Lyme disease, sinus node dysfunction also seems to respond well to antibiotic therapy as was demonstrated in this case. The simultaneous presence of accelerated junctional rhythm is consistent with inflammation of $\mathrm{AV}$ node and provides another clue to the diagnosis.

Given the paucity of cases of sinus node dysfunction, especially with accelerated junctional rhythm in the setting of Lyme disease, we present this case to enhance the overall understanding of various presentations of Lyme carditis.

\section{Conclusion:}

Accurate diagnosis and monitoring of the clinical progression of sinus node dysfunction in the setting of Lyme carditis is essential given the likelihood of recovery with conservative management with antibiotics and observation, even if temporary pacemaker is required. Permanent pacemaker placement should be delayed to allow recovery even if active fixation permanent pacing lead, with an externalized pacemaker that can be reused is needed $[7-8,10]$. When comparing case reports, it is also very important to assess levels of block as our patient had normal AV conduction with every atrial premature complex or with a sinus beat that intermittently manifested two days after starting therapy. The accelerated junctional rhythm was felt to be due to inflammation of the AV node due to Lyme carditis. Our patient recovered quickly and was only kept in the hospital for 4 days until the junctional escape rhythm was overtaken by the recovered sinus node. However, other case reports have shown persistent AV block despite antibiotic therapy without need for a pacemaker [8].

\section{Conflict of Interest Disclosures:}

Dr. Rosenfeld: Fellowship support from: Boston Scientific, Medtronic, and St Jude Medical

Dr. Bhatt: Honoraria: St. Jude Medical, Biotronik, Zoll

\section{References}

1. Stanek G, Strle F.. Lyme borreliosis. Lancet. 2003 Nov 15;362(9396):1639-1647. PUMED

2. Shapiro ED. Clinical practice. Lyme disease. N Engl J Med. 2014 May 1;370(18):1724-1731. PUMED

3. Fish AE, Pride YB, Pinto DS. Lyme carditis. Infect Dis Clin North Am. 2008 Jun;22(2):275-288. PUMED

4. Lelovas P, Dontas I, Bassiakou E, Xanthos T. Cardiac implications of Lyme disease, diagnosis and therapeutic approach. Int J Cardiol. 2008; 129:15-21. PUMED

5. Pinto DS. Cardiac manifestations of Lyme disease. Medical Clinics of North America. 2002; 86:285-296. PUMED

6. Franck H. Wollschlager H. Lyme carditis and symptomatic sinus node dysfunction. Z Kardiol. 2003; 92:1029-1032. PUMED

7. Oktay AA, Dib SR, Friedman H. Sinus pause in association with Lyme carditis. Tex Heart Inst J. 2015; 42:248-250. PUMED

8. Bartunek P, Nemec J, Mrazek V, Gorican K, Zapletalova J. Borrelia burgdorferi as a cause of sick sinus syndrome. Cas Lek Cesk. 1993; 135:729-731. PUMED

9. Greenberg YJ, Brennan JJ, Rosenfeld LE. Lyme myocarditis presenting as fascicular tachycardia with underlying complete heart block. J Cardiovasc Electrophysiol. 1997 Mar;8(3):323324. PUMED

10. Rosenfeld LE. Temporary permanent or permanent temporary pacing? Pacing Clin Electrophysiol. 2011 Jun;34(6):670-671.doi: 10.1111/j.1540-8159.2011.03086.x. Epub 2011 Mar 31. PUMED

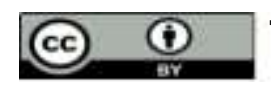

This work is licensed under Creative Commons Attribution 4.0 License

To Submit Your Article Click Here: Submit Article

DOI:10.31579/2641-0419/106
Ready to submit your research? Choose Auctores and benefit from:

$$
\begin{aligned}
& \text { * fast, convenient online submission } \\
& \text { *igorous peer review by experienced research in your field } \\
& \text { *apid publication on acceptance } \\
& \text { * authors retain copyrights } \\
& \text { * unique DOI for all articles } \\
& \text { immediate, unrestricted online access }
\end{aligned}
$$

At Auctores, research is always in progress.

Learn more www.auctoresonline.org/journals/clinical-cardiology-andcardiovascular-interventions 